\title{
Local incompressibility of fractional quantum Hall states at a filling factor of $3 / 2$
}

\author{
L. V. Kulik, ${ }^{1,2}$ V. A. Kuznetsov $\odot, 2,1,{ }^{*}$ A. S. Zhuravlev, ${ }^{1}$ V. Umansky $\odot,{ }^{3}$ and I. V. Kukushkin ${ }^{1,2}$ \\ ${ }^{1}$ Institute of Solid State Physics Russian Academy of Sciences Chernogolovka, Moscow District, \\ 2 Academician Ossipyan Street, 142432, Russia \\ ${ }^{2}$ National Research University Higher School of Economics, Moscow, 20 Myasnitskaya Street, 101000, Russia \\ ${ }^{3}$ Braun Center for Submicron Research, Weizmann Institute of Science, 234 Herzl Street, POB 26, Rehovot 76100, Israel
}

(Received 15 November 2019; revised 13 March 2020; accepted 20 May 2020; published 22 July 2020)

\begin{abstract}
We studied neutral excitations in a two-dimensional electron gas with an orbital momentum $\Delta M=1$ and spin projection over the magnetic field axis $\Delta S_{z}=1$ in the vicinity of a filling factor of $3 / 2$. It is shown that the $3 / 2$ state is a singular point in the filling factor dependence of the spin ordering of the two-dimensional electron gas. In the vicinity of $v=3 / 2$, a significant increase in the relaxation time ( $\tau=13 \mu \mathrm{s})$ for the excitations to the ground state is exhibited even though the number of vacancies in the lowest energy level is macroscopically large. The decrease in the relaxation rate is related to the spin texture transformation in the ground state induced by spin flips and electron density rearrangement. Based on the experimental data we believe that the $3 / 2$ state is an example of locally incompressible fractional quantum Hall state.
\end{abstract}

DOI: 10.1103/PhysRevResearch.2.033123

\section{INTRODUCTION}

The interest in excitations in a two-dimensional electron gas (2DEG) placed in a quantizing magnetic field is due to their use in a number of fundamental applications. These applications are based on the progress of topological quantum calculations [1,2], the involvement of anyons (quasiparticles with neither Bose nor Fermi statistics, and at some filling factors, even being non-Abelian) to describe the physical properties of 2DEG [3,4], as well as the discovery of new condensed states that have no obvious analogs in three-dimensional systems [5]. Non-Abelian excitations are discussed in relation to fractional quantum Hall states with even denominators. A topological quantum computer, an extremely attractive idea for computation protected from mistakes caused by quantum state decoherence, can be realized using non-Abelian anyons [6]. The first fractional quantum Hall state of this type, $5 / 2$, was found in experiments on magnetotransport in highmobility two-dimensional GaAs/AlGaAs-based heterostructures $[7,8]$. More stable at first glance, fractional quantum Hall state at $v=3 / 2$ was not observed in GaAs until recently $[9,10]$. Here we do not consider intricate electron systems with complementary degrees of freedom: a layer index in double electron layers or a size-quantized subzone in wide quantum wells. The 3/2 state in $\mathrm{GaAs} / \mathrm{AlGaAs}$ heterostructures was described as similar to the $1 / 2$ state of composite fermions

\footnotetext{
*Author to whom correspondence should be addressed: volod_kuzn@issp.ac.ru
}

Published by the American Physical Society under the terms of the Creative Commons Attribution 4.0 International license. Further distribution of this work must maintain attribution to the author(s) and the published article's title, journal citation, and DOI.
[11,12], i.e., a partially spin-polarized state of composite fermions with a zero effective magnetic field and a hierarchy of fractional states originating in the $3 / 2$ state as a hierarchy of integer quantum Hall states of composite fermions $[13,14]$. Here lies the difference between the $1 / 2$ and $3 / 2$ states, on the one hand, and the $5 / 2$ state, on the other hand, since the $5 / 2$ state is regarded as fully spin polarized [15], which is a necessary condition for the formation of anti-Pfaffian and similar type ground states [16-18]. This physical view was qualitatively confirmed in magnetotransport studies of the fractional quantum Hall effect in novel highly mobile two-dimensional electron systems based on $\mathrm{ZnO} / \mathrm{MgZnO}$ heterostructures. Significantly higher Zeeman splitting in $\mathrm{ZnO}$ makes it possible to engineer a fully spin-polarized $3 / 2$ state. This can be done by tilting the external magnetic field from the perpendicular to the heterostructure surface. As the electron system becomes spin polarized, the Hall resistance exhibits the formation of a $3 / 2$ plateau [19], which makes the $3 / 2$ state potentially interesting for topological quantum calculations.

All these studies deal with the edge state transport of fractional Hall insulators. The existing theoretical models point to a correlation between the transport properties of bulk and edge states in Hall insulators. Therefore, the transport characteristics of a Hall insulator allow for making far-reaching conclusions on the nature of the bulk ground state [20]. Unlike in Hall insulators, transport measurements give much less information on the nature of the conducting states in fractional Hall conductors. This advances interest in the direct investigation of the bulk properties of conducting fractional quantum Hall states. We have developed optical techniques to probe bulk states of 2DEG in the quantum Hall regime, namely, resonant reflection (RR), photoinduced resonant reflection (PRR), and photoinduced photoluminescence, which can be used for the investigation of bulk states in Hall insulators and in Hall conductors [21]. The reflection 
signal in the quantum Hall regime appears in presence of vacancies in the zero Landau level of the conduction band. A laser tuned to the transition from the zero Landau level of heavy holes in valence band to the zero Landau level of conduction band can be absorbed and then reemitted back with the same momentum and energy. This process is called resonant reflection (RR). With an additional pump laser that promotes the electrons to higher Landau levels, one can expect the formation of nonequilibrium dark magnetoexciton ensemble. This ensemble consists of magnetoexcitons with spin and spin projection to the magnetic field axis: $\Delta S=1$, $\Delta S_{z}=-1$. The respective change in RR, namely reflection in the presence of the nonequilibrium ensemble is called the photoinduced resonance reflection (PRR). Thus, the essence of PRR technique reduces to the optical formation and detection of nonequilibrium ensembles of magnetoexcitons. Such excitations cannot relax directly to the 2DEG ground state with photon or phonon emission if the lowest energy spin Landau sublevel (up-spin sublevel) is completely occupied, and the spin-orbital interaction is small, as, for example, in the conduction band of GaAs, (this is discussed in detail in Ref. [22]). Such a relaxation process is impossible, for instance, at integer filling factor $v=2$, which reflects the incompressibility of the electron gas in the lowest energy spin sublevel of Landau level. This also leads to the formation of charged three-particle complexes (plasmarons and trions) [29,31].

As the filling factor is reduced from $v=2$, the $2 \mathrm{DEG}$ is depolarized and there are no principle restrictions on the relaxation of photoexcited electrons from both spin sublevels of the first Landau level $\left(M=1, S_{z}= \pm 1 / 2\right)$ to the ground state [23]. As a result, $\Delta M=1, \Delta S_{z}=1$ collective excitations must be unstable. However, we observe a significant increase of the relaxation time for such excitations in the $3 / 2$ fractional conducting state. The $3 / 2$ state in GaAs/AlGaAs heterostructures appears to be a much more complex physical object than has been considered. Based on our experimental results we claim that the $3 / 2$ state is an ordered spin configuration (spin texture) in which spin-flip excitations with a change of orbital momentum are accompanied by the spintexture transformation. This texture transformation forbids the relaxation of the excited electron even though the mean spin polarization through the sample is much less than unity [15], i.e., there is a macroscopically large number of vacant places in the ground state of the $2 \mathrm{DEG}$ suitable for the relaxation of the excited electron spin. In other words, we are not dealing with the incompressibility of the whole 2DEG as in the case of filling factor 2 , but with a local incompressibility: the inability to return the excited electron to the ground state in close proximity to the excited electron itself.

\section{EXPERIMENT}

\section{A. Samples}

The experiment was performed using high-quality heterostructures with symmetrically doped single GaAs/ AlGaAs/GaAs quantum well with electron concentration in the two-dimensional channel from $1.8 \times 10^{11} \mathrm{~cm}^{-2}$ to $2.6 \times 10^{11} \mathrm{~cm}^{-2}$ and dark mobility more than $1.5 / 4.0 \times$
$10^{7} \mathrm{~cm}^{2} / \mathrm{V} \mathrm{s}$. In addition, symmetric doping was required to minimize the penetration of the electron wave function into the barrier of the quantum well. The quantum well widths were $30 / 40 \mathrm{~nm}$. To be noted, we were unable to observe the discussed effects in two samples with the electron density of $3 \times 10^{11} \mathrm{~cm}^{-2}$ and $3.5 \times 10^{11} \mathrm{~cm}^{-2}$. Since there is a qualitative agreement between the experimental results for different heterostructures, we focus here on the results obtained for heterostructures with a quantum well width of $31 \mathrm{~nm}$ and a concentration of $1.8 / 2.1 \times 10^{11} \mathrm{~cm}^{-2}$.

\section{B. Experimental setup}

The samples were placed into a pumped cryostat with liquid ${ }^{3} \mathrm{He}$, which in turn was put into a cryostat with a superconducting solenoid. Optical measurements were made in the temperature range of $0.45-1.5 \mathrm{~K}$.

In the experiments, we used a double optical fiber technique. One fiber was used for the CW resonant and nonresonant photoexcitation of the 2DEG. The size of the photoexcited spot was $1 \mathrm{~mm}^{2}$. The other fiber was used to collect the resonant reflection signal and the photoluminescence signal from the photoexcitation spot. Output light was analyzed by a grating spectrometer with a cooled CCD camera. A broadband laser diode (excitation wavelength $780 \mathrm{~nm}$ and spectral width $10 \mathrm{~nm}$ ) was used as an optical source to form a nonequilibrium spin excitation ensemble and a photoluminescence signal. TOptica tunable diode laser with a spectral bandwidth of $20 \mathrm{kHz}$ was used to measure resonant reflection. The relaxation time was measured in the time range of $1 / 1000 \mu \mathrm{s}$ by modulating the laser diode current with a pulse generator. The photoexcitation density varied within $1 / 100 \mu \mathrm{W} / \mathrm{mm}$, which did not cause the overheating of the 2DEG. PRR per se does not involve the formation of excitations, yet, the 2DEG resonant reflection and photoluminescence signals overlap spectrally. For measurements of the photoluminescence spectra, the resonant excitation was switched off. The parasitic reflection from the sample surface was filtered by crossed wire-grid linear polarizers placed between the optical fibers and the sample. Both resonant reflection and luminescence have circular polarization and pass through the linear polarizer.

\section{RESULTS AND DISCUSSION}

In Fig. 1, there are examples of the spectra of resonant reflection and photoinduced resonant reflection spectra from the zero Landau level states of the 2DEG measured in the quantum Hall conductor in the vicinity of filling factor $v=$ $3 / 2$. At filling factors lower than and above $3 / 2$ (1.3 and 1.7) the RR and PRR signals coincide. This is reasonable as the 2DEG is only partially spin polarized, and there is a macroscopic number of vacant places for the relaxation of the excited electron to the zero Landau level without spin flip and with the emission of a cyclotron energy photon. In this case the 2DEG turns into an excited spin state from which it just as easily relaxes to the ground state with the emission of an electron spin resonance photon. The relaxation time lies in the nanosecond range [24,25], so it is not possible to accumulate any considerable number of excitations with such 


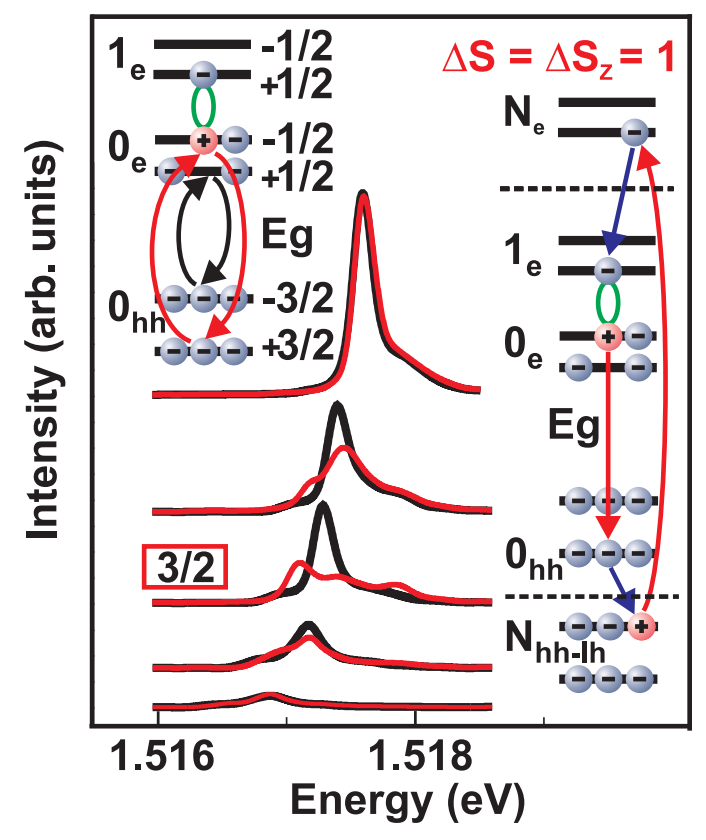

FIG. 1. Resonant reflection (RR) (black line) and photoinduced resonant reflection (PRR) spectra (red line) at different filling factors $(1.3,1.45,1.5,1.55,1.7$ from top to bottom) of the 2DEG in the vicinity of $(v=3 / 2)$ measured at the photoexcitation level $100 \mu \mathrm{W} / \mathrm{mm}$ and temperature $T=0.45 \mathrm{~K}$. On the right, a diagram of photoexcitation of a nonequilibrium excitation with spin and orbital quantum number 1 is shown including: (i) optical transitions from a state in a Landau level of valence band $\left(N_{h h-l h}\right.$, where hh and $\mathrm{lh}$ are heavy and light holes) to a state in a Landau level of the conduction band; (ii) hole spin-flip relaxation in the valence band due to the large spin-orbital interaction in the GaAs valence band to the lowest energy state in the zero Landau level of heavy holes $\left(0_{h h}\right)$; (iii) recombination of an electron in the zero Landau level of the conduction band $\left(0_{e}\right)$ and the hole in the zero Landau level of the heavy hole in the valence band $\left(0_{h h}\right)$ resulting to a Fermi hole in the upper spin state of the zero Landau level of the conduction band; (iv) relaxation of the excited electron with appropriate spin to the lowest spin state of the first Landau level (spin-orbital interaction in the GaAs conduction band is weak); and finally (v) binding of the excited electron and the Fermi hole. Examples of the RR and PRR processes are shown on the left side of the diagram.

short lifetimes and the photoexcitation density used in the experiment.

With a decreasing 2DEG filling factor the number of unoccupied places at the zero Landau level increases and the RR and PRR signals grow accordingly. However, of principal interest is not the absolute value of the reflection signal but the distribution of vacant sites between the two spin states in the zero Landau level ( $g$ factor is negative in GaAs): $\kappa=$ $(I \downarrow-I \uparrow) /(I \downarrow+I \uparrow)$ (Fig. 2). $I \downarrow$ and $I \uparrow$ are amplitudes of the resonant reflection signal from spin-down and spin-up sublevels of the zero Landau level. These amplitudes were calculated by fitting of the experimental RR spectrum. The ratio between the empty places in the upper and lower spin Landau sublevels decreases monotonically from filling factor 1.6 to filling factor 1 according to the RR signal. At filling factor 1, the lower spin Landau sublevel is occupied, and the upper spin Landau sublevel is empty (Hall ferromagnet).

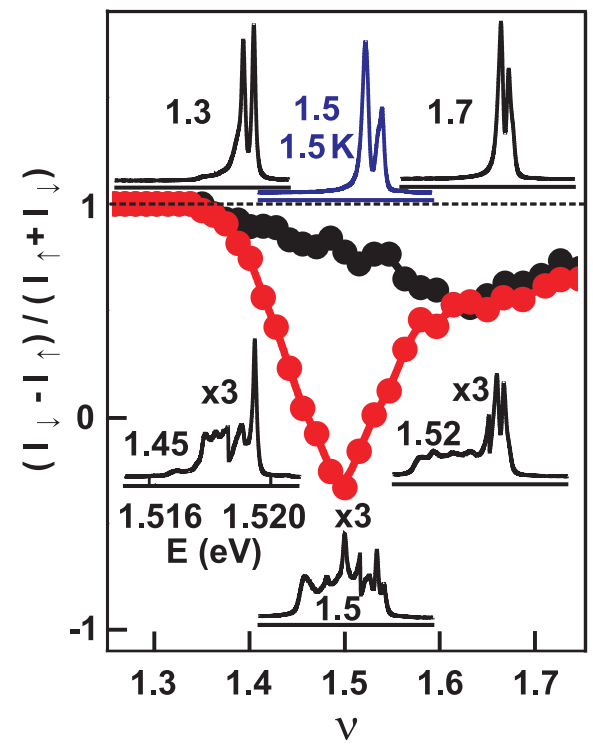

FIG. 2. $\alpha=(I \downarrow-I \uparrow) /(I \downarrow+I \uparrow)$ measured from the integral intensity of resonant reflection (RR) (black dots) and photoinduced resonant reflection (PRR) (red dots) lines as a function of filling factor. The insets show photoinduced photoluminescence spectra measured in the same energy interval at the temperature of $0.45 \mathrm{~K}$ (black lines) and different filling factors. There is a spectrum measured at the temperature of $1.5 \mathrm{~K}$ at the filling factor $v=3 / 2$ (blue line). Spectra at $v<1.3$ and $v>1.6$ as well as the spectrum at $1.5 \mathrm{~K}$ consist of two lines corresponding to the recombination of valence band holes $\left(0_{h h}\right)$ with the conduction band $\left(0_{e}\right)$. Both transitions are active in two polarizations of the emitted light. When the nonequilibrium ensemble of excitations with orbital and spin quantum number 1 is created at $v>1.3$ and $v<1.6$ and a temperature $T<0.8 \mathrm{~K}$ a variety of new lines appears in the photoluminescence spectra.

Therefore, the observed PRR pattern of the empty place distribution between two spin sublevels is natural.

Surprisingly, the reflection signal under nonresonant exitation is nonzero in the vicinity of filling factor $3 / 2$ due to the creation of nonequilibrium spin excitations (Fig. 2). The number of unoccupied states in the lower spin Landau level increases dramatically during the formation of a nonequilibrium spin excitation ensemble. Simultaneously, the lifetime of nonequilibrium spin excitations reaches values over $10 \mu \mathrm{s}$, which is only an order of magnitude less than the lifetime of analogous excitations in the Hall insulator at filling factor 2 (Fig. 3). At filling factor 2, the $2 \mathrm{DEG}$ is in the incompressible integer quantum Hall state with a large energy gap at the Fermi level, while the $3 / 2$ state is a Hall conductor with no energy gap at the Fermi level at all. Even in the incompressible Hall ferromagnet state at $v=1$, the relaxation of excitations with orbital momentum $\Delta M$ and spin projection $S_{z}=1$ proceeds much faster than at filling factor $3 / 2$. It occurs despite the number of empty places at the lower spin sublevel in the Hall ferromagnet being much less than in the $3 / 2$ state (yet it is not exactly equal to zero at experimentally achieved temperatures [26]).

It is possible to roughly estimate the nonequilibrium density of excitations with $\Delta M=1$ and $\Delta S_{z}=1$ at filling factor $3 / 2$ as we know of the dependencies of the nonequilibrium 


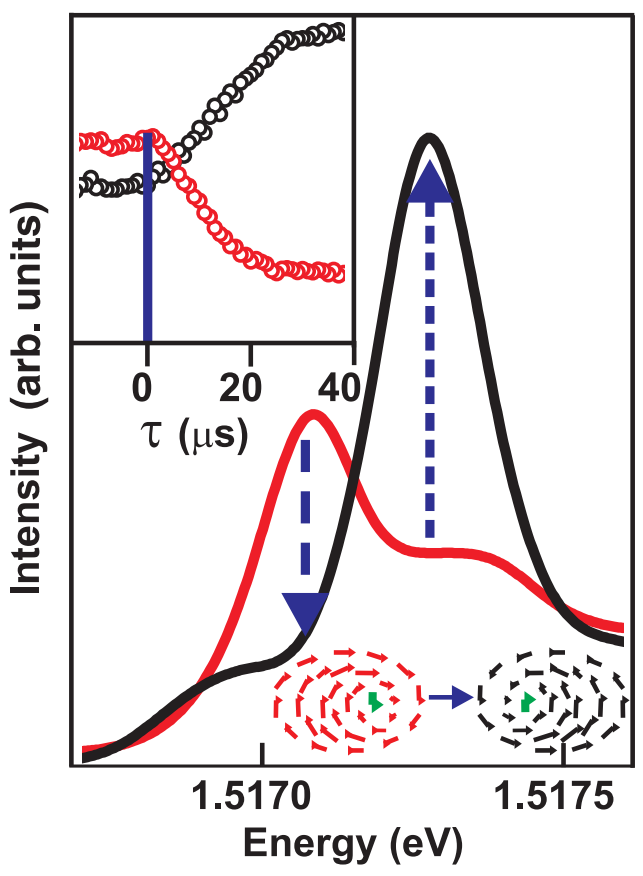

FIG. 3. Resonant reflection (RR) (black line) and photoinduced resonant reflection (PRR) spectra (red line) measured at filling factor $3 / 2$ at the same experimental parameters as in Fig. 1. The inset shows the relaxation dynamics of the PRR spectra after photoexcitation switch off. Below is a schematic illustration of spin texture transformation associated with photoexcitation-induced changes of the spin and orbital quantum numbers of the electron system.

excitation density on the photoexcitation density and on the excitation relaxation time obtained from the studies of the Hall insulator $v=2[21,22]$. At the maximum allowed photoexcitation density causing no overheating of the electron system, $100 \mu \mathrm{W} / \mathrm{mm}^{2}$, this value is about $1 \%$ of the total magnetic flux quanta at a single Landau level. If we assume that excitation effectiveness does not depend on filling factor then excitation density depends only on lifetime. For the filling factor $v=2$, maximum of the excitation density is 0.1 of $\mathrm{LL}$, and lifetime is $100 \mu \mathrm{s}$. At $v=3 / 2$ lifetime is $10 \mu \mathrm{s}$, then excitation density is about 0.01 of the LL.

From Fig. 2 the rough estimation of spin flips in the 2DEG ground state is about 27 per excited electron; i.e., the formation of a single spin excitation with $\Delta M=1$ and $\Delta S_{z}=1$ is followed by the rearrangement of the $\sim 27$ spins in the ground state. Assuming that PRR intensity $I$ linearly depends on excitation density $n$, we can estimate number of excited electrons in one excitation. Thus, dimensionless degree of spin polarization $\kappa=\frac{I_{\downarrow}-I_{\uparrow}}{I_{\downarrow}+I_{\uparrow}}=\frac{n_{\downarrow}-n_{\uparrow}}{n_{\downarrow}+n_{\uparrow}}$ In the equlibrium, $\kappa=0.79$. Under photoexcitation, $\kappa=-0.34$. This gives us $\Delta n=0.28$. Knowing excitation density, we get 27 electrons per one excitation as an estimate. Accordingly, relaxation to the ground state should also accompany $\sim 27$ spin flips and the spin-texture rearrangement to the ground state. Despite the metallic conductivity of the $2 \mathrm{DEG}$ at $v=3 / 2$, we claim that the electrons are united into large spin textures (much larger than those existing in the vicinity of Hall ferromagnet $v=1$ $[27,28])$. As a consequence, a physical phenomenon similar to incompressibility of the 2DEG emerges in the proximity of

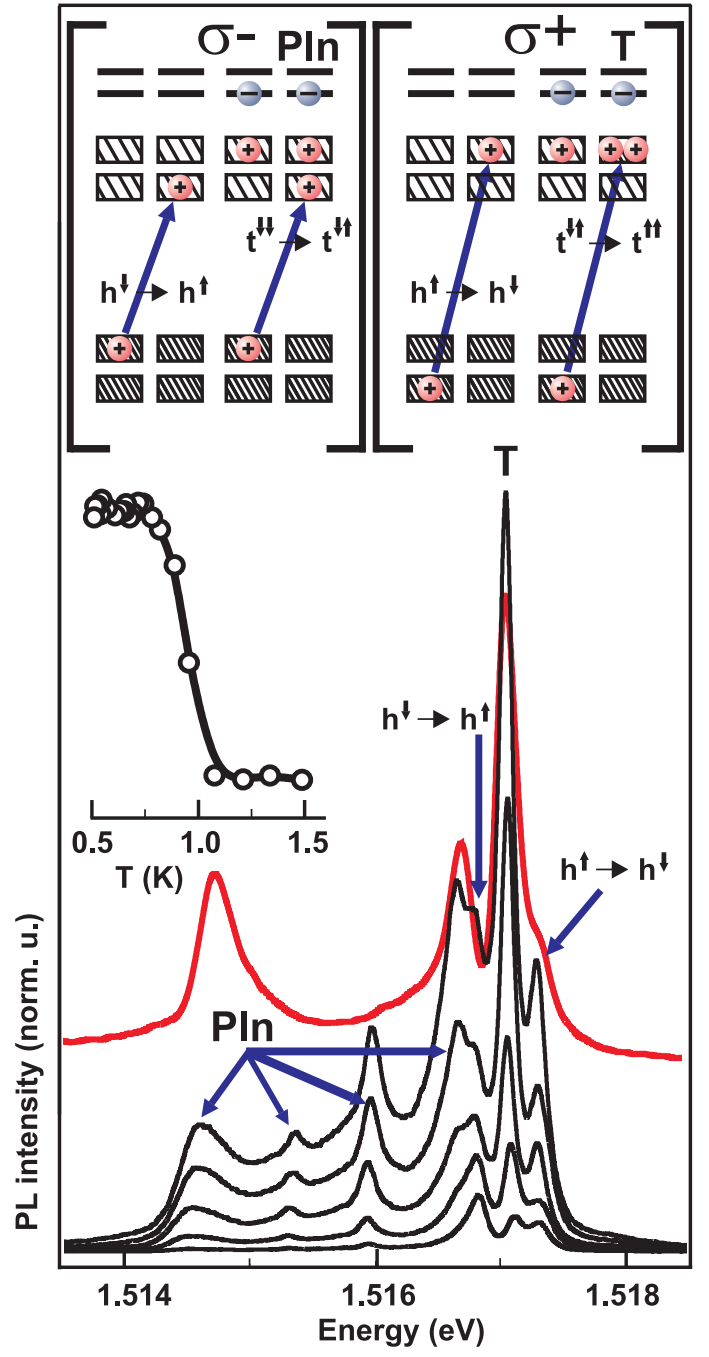

FIG. 4. Above shown is a diagram of optical photoluminescence transitions in two polarizations of the emitted photon in the absence and presence of excitation with spin and orbital quantum number 1 . Below shown are photoinduced photoluminescence spectra at filling factor $3 / 2$ measured at various photoexcitation levels that are 1 , $1.5,3,6$, and 16 times less than the maximal photoexcitation level $100 \mu \mathrm{W} / \mathrm{mm}$ (black solid lines). For comparison, a photoinduced photoluminescence spectrum measured at filling factor 2 at an excitation level of $10 \mu \mathrm{W} / \mathrm{mm}$ (red solid line) is presented. The energy scales are equal for black and red lines. The inset shows the ratio of the integral intensity of the plasmaron line to the integral of photoluminescence signal.

the photoexcited electron (local incompressibility). That is the reason for huge experimental relaxation times for the excited electrons.

Not the least important criterion of formation of a nonequilibrium excitation with $\Delta M=1$ and $\Delta S_{z}=1$ is the appearance of recombination lines of three-particle complexes composed of a photoexcited hole and nonequilibrium excitation itself in the 2DEG photoluminescence spectrum. In the carefully studied case of a Hall insulator at $v=2$, there are two types of such complexes defined by the spin of the photoexcited hole (Fig. 4) [29]. After recombination, the photoexcited hole turns to a vacancy in the filled Landau 
level of the conduction band (a Fermi hole). There are two bound three-particle complexes built of the nonequilibrium excitation and the Fermi hole. One hole appears because of the recombination and another is confined in the nonequilibrium excitation. If the spins of two Fermi holes form a spin triplet, the three-particle complex is a trion. If the spins of two Fermi holes form a spin singlet, the three-particle complex is a plasmaron since the excited electron may recombine with one of the Fermi holes transferring energy and momentum to a new electron-hole pair (plasma oscillation). The plasmaron can be regarded as a magnetoplasmon [30] bound to an extra Fermi hole.

The intensity of the trion line accounts for the whole density of the nonequilibrium excitations with $\Delta M=1$ and $\Delta S_{z}=1$ in the excitation spot. Plasmaron photoluminescence contains information on the total number of excitations as well as on the plasmarons spectral density that yields, in turn, the density distribution of nonequilibrium excitations in the momentum space before they bind to the Fermi hole [29,31]. The model is only approximately valid for the fractional ground state. Interaction of three-particle complexes with surrounding Fermi holes or spin textures as in the case of $v=3 / 2$ must be taken into account.

The trion and plasmaron lines are observed in the vicinity of filling factor $3 / 2$ after the formation of the nonequilibrium ensembles of excitations with orbital and spin quantum number 1 (Fig. 2). The trion line at filling factor $3 / 2$ is similar to that at filling factor 2. Yet, the plasmaron line is significantly modified. The plasmaron spectrum exhibits extra maxima that are absent in the spectrum of plasmaron obtained at filling factor 2 (the lines in the plasmaron spectrum at filling factor 2 correspond to the high-density states on the dispersion curve of magnetoplasmons: maxima and minima [32]). An increase in photoexcitation power density changes the ratio between one- and three-particle recombination channels. However, the plasmaron spectrum at $v=3 / 2$ does not display any changes. That means we observe a single-particle distribution of magnetoplasmons in the momentum space, and this is radically different from the case of $v=2$ [29], where a change in excitation density is accompanied with the redistribution of excitations in the momentum space. With increasing temperature, the photoluminescence of three-particle complexes and the PRR signal disappear thresholdwise simultaneously (Figs. 2 and 4). The characteristic transition temperature is $0.8 \mathrm{~K}$ that is much higher than the temperature of composite fermion formation. Yet, it is twice less than the Zeeman activation gap $(1.6 \mathrm{~K})$ pointing out on the spin nature of the observed phenomenon.

Our experiments qualitatively support the transport measurements of a spatially confined 2DEG at $v=3 / 2$ [10]. The 2DEG incompressibility revealed by Zhang et al. may occur due to the local incompressibility of the $3 / 2$ fractional state and commensurability of the constriction in the confining potential with the size of one or few spin textures. The confined geometry was proposed by Wen as a powerful tool to probe the internal topological order of $\mathrm{FQH}$ states [20]. One may view the charge current at $v=3 / 2$ being performed not by separate electrons but spin textures. When the size of the confined region in the Hall conductor becomes comparable with the spin texture size, the transport in the bulk of the 2DEG terminates, and there is only the edge channel transport. As a result, the $R_{x y}$ is quantized at $v=3 / 2$ in the sample with lateral confinement. The spatially confined $v=3 / 2$ state is an example of locally incompressible fractional quantum Hall state that is neither Laughlin liquid nor integer quantum Hall state of composite fermions.

\section{CONCLUSION}

Optical techniques were used to investigate excited states with orbital momentum $\Delta M=1$ and $\Delta S_{z}=1$ near fractional filling factor $3 / 2$ in a $2 D E G$. A huge increase in the excitation relaxation time $(\tau=13 \mu \mathrm{s})$ was discovered. We claim that the spin field is spatially redistributed owing to the existence of an excited electron and a corresponding vacancy. The electrons are united in spin textures with the different spin configurations of the excited and ground states.

\section{ACKNOWLEDGMENTS}

The work was supported by Russian Science Foundation, Grant No. 18-12-00246.

L.V.K., V.A.K., A.S.Z., and I.V.K. designed experiments. V.U. manufactured samples. V.A.K. and A.S.Z. performed experiments. A.S.Z. processed raw data, L.V.K. prepared figures. L.V.K. and V.A.K. wrote the manuscript. All authors participated in the results discussion. The authors declare no competing financial interests. Data are available from the corresponding author upon request.
[1] W. M. Witzel and S. Das Sarma, Quantum theory for electron spin decoherence induced by nuclear spin dynamics in semiconductor quantum computer architectures: Spectral diffusion of localized electron spins in the nuclear solid-state environment, Phys. Rev. B 74, 035322 (2006).

[2] A. Yu. Kitaev, Fault-tolerant quantum computation by anyons, Ann. Phys. (NY) 303, 2 (2003).

[3] F. Wilczek, Quantum Mechanics of Fractional-Spin Particles, Phys. Rev. Lett. 49, 957 (1982).

[4] F. Wilczek, Fractional Statistics and Anyon Superconductivity (World Scientific, Singapore, 1990).
[5] R. B. Laughlin, Anomalous Quantum Hall Effect: An Incompressible Quantum Fluid with Fractionally Charged Excitations, Phys. Rev. Lett. 50, 1395 (1983).

[6] C. Nayak, S. H. Simon, A. Stern, M. Freedman, and S. Das Sarma, Non-abelian anyons and topological quantum computation, Rev. Mod. Phys. 80, 1083 (2008).

[7] R. Willett, J. P. Eisenstein, H. L. Störmer, D. C. Tsui, A. C. Gossard, and J. H. English, Observation of an Even-Denominator Quantum Number in the Fractional Quantum Hall Effect, Phys. Rev. Lett. 59, 1776 (1987). 
[8] R. L. Willett, L. N. Pfeiffer, and K. W. West, Measurement of filling factor $5 / 2$ quasiparticle interference with observation of charge e $/ 4$ and e/2 period oscillations, Proc. Nat. Acad. Sci. 106, 8853 (2009).

[9] Y. Zhang, Waves, particles, and interactions in reduced dimensions, Ph.D. thesis, Harvard University, Cambridge, Massachusetts, 2009, p. 125, Fig. 9.9.

[10] H. Fu, Y. Wu, R. Zhang, J. Sun, P. Shan, P. Wang, Z. Zhu, L. N. Pfeiffer, K. W. West, H. Liu, X. C. Xie, and X. Lin, 3/2 fractional quantum Hall plateau in confined two-dimensional electron gas, Nat. Commun. 10, 4351 (2019).

[11] B. I. Halperin, Theory of the quantized Hall conductance, Helv. Phys. Acta 56, 75 (1983).

[12] B. I. Halperin, P. A. Lee, and N. Read, Theory of the half-filled Landau level, Phys. Rev. B 47, 7312 (1993).

[13] R. R. Du, A. S. Yeh, H. L. Stormer, D. C. Tsui, L. N. Pfeiffer, and K. W. West, Fractional Quantum Hall Effect Around $v=$ 3/2: Composite Fermions with a Spin, Phys. Rev. Lett. 75, 3926 (1995)

[14] G. Murthy and R. Shankar, Hamiltonian theories of the fractional quantum Hall effect, Rev. Mod. Phys. 75, 1101 (2003).

[15] L. Tiemann, G. Gamez, N. Kumada, and K. Muraki, Unraveling the spin polarization of the $v=5 / 2$ fractional quantum Hall state, Science 335, 828 (2012).

[16] G. Moore and N. Read, Nonabelions in the fractional quantum Hall effect, Nucl. Phys. B 360, 362 (1991).

[17] S.-S. Lee, S. Ryu, C. Nayak, and M. P. A. Fisher, Particle-Hole Symmetry and the $v=5 / 2$ Quantum Hall State, Phys. Rev. Lett. 99, 236807 (2007).

[18] D. Thanh Son, Is the Composite Fermion a Dirac Particle? Phys. Rev. X 5, 031027 (2015).

[19] J. Falson, D. Maryenko, B. Friess, D. Zhang, Y. Kozuka, A. Tsukazaki, J. H. Smet, and M. Kawasaki, Even-denominator fractional quantum Hall physics in ZnO, Nat. Phys. 11, 347 (2015).

[20] X.-G. Wen, Theory of the edge states in fractional quantum Hall effects, Int. J. Mod. Phys. B 06, 1711 (1992).

[21] L. V. Kulik, A. S. Zhuravlev, S. Dickmann, A. V. Gorbunov, V. B. Timofeev, I. V. Kukushkin, and S. Schmult, Magnetofermionic condensate in two dimensions, Nat. Commun. 7, 13499 (2016).
[22] L. V. Kulik, A. V. Gorbunov, A. S. Zhuravlev, V. B. Timofeev, S. Dickmann, and I. V. Kukushkin, Super-long life time for 2D cyclotron spin-flip excitons, Sci. Rep. 5, 10354 (2015).

[23] E. H. Aifer, B. B. Goldberg, and D. A. Broido, Evidence of Skyrmion Excitations about $v=1$ in $n$-Modulation-Doped Single Quantum Wells by Interband Optical Transmission, Phys. Rev. Lett. 76, 680 (1996).

[24] Q. Zhang, T. Arikawa, E. Kato, J. L. Reno, W. Pan, J. D. Watson, M. J. Manfra, M. A. Zudov, M. Tokman, M Erukhimova, A. Belyanin, and J. Kono, Superradiant Decay of Cyclotron Resonance of Two-Dimensional Electron Gases, Phys. Rev. Lett. 113, 047601 (2014).

[25] A. S. Zhuravlev, S. Dickmann, L. V. Kulik, and I. V. Kukushkin, Slow spin relaxation in a quantum Hall ferromagnet state, Phys. Rev. B 89, 161301(R) (2014).

[26] P. Plochocka, J. M. Schneider, D. K. Maude, M. Potemski, M. Rappaport, V. Umansky, I. Bar-Joseph, J. G. Groshaus, Y. Gallais, and A. Pinczuk, Optical Absorption to Probe the Quantum Hall Ferromagnet at Filling Factor $v=1$, Phys. Rev. Lett. 102, 126806 (2009).

[27] S. L. Sondhi, A. Karlhede, S. A. Kivelson, and E. H. Rezayi, Skyrmions and the crossover from the integer to fractional quantum Hall effect at small Zeeman energies, Phys. Rev. B 47, 16419 (1993).

[28] H. A. Fertig, L. Brey, R. Côté, and A. H. MacDonald, Charged spin-texture excitations and the Hartree-Fock approximation in the quantum Hall effect, Phys. Rev. B 50, 11018 (1994).

[29] A. S. Zhuravlev, V. A. Kuznetsov, L. V. Kulik, V. E. Bisti, V. E. Kirpichev, I. V. Kukushkin, and S. Schmult, Artificially Constructed Plasmarons and Plasmon-Exciton Molecules in 2D Metals, Phys. Rev. Lett. 117, 196802 (2016).

[30] Y. A. Bychkov, S. V. Iordanskii, and G. M. Eliashberg, Twodimensional electrons in a strong magnetic field, JETP Lett. 33, 143 (1981).

[31] V. A. Kuznetsov, L. V. Kulik, M. D. Velikanov, A. S. Zhuravlev, A. V. Gorbunov, S. Schmult, and I. V. Kukushkin, Threeparticle electron-hole complexes in two-dimensional electron systems, Phys. Rev. B 98, 205303 (2018).

[32] C. Kallin and B. I. Halperin, Excitations from a filled Landau level in the two-dimensional electron gas, Phys. Rev. B 30, 5655 (1984). 